TRANSACTIONS OF THE

AMERICAN MATHEMATICAL SOCIETY

Volume 363, Number 1, January 2011, Pages 95-108

S 0002-9947(2010)05260-2

Article electronically published on August 11, 2010

\title{
FRAMES GENERATED BY ACTIONS OF COUNTABLE DISCRETE GROUPS
}

\author{
KJETIL RØYSLAND
}

\begin{abstract}
We consider dual frames generated by actions of countable discrete groups on a Hilbert space. Module frames in a class of modules over a group algebra are shown to coincide with a class of ordinary frames in a representation of the group. This has applications to shift-invariant spaces and wavelet theory. One of the main findings in this paper is that whenever a shift-invariant subspace in $L^{2}\left(\mathbb{R}^{n}\right)$ has compactly supported dual frame generators, then it also has compactly supported bi-orthogonal generators. The crucial part in the proof is a theorem by Swan that states that every finitely generated projective module over the Laurent polynomials in $n$ variables is free.
\end{abstract}

\section{INTRODUCTION}

In several branches of applied mathematics one seeks suitable decompositions of a Hilbert space $\mathcal{H}$. This could, for instance, be done using an orthonormal basis, as is often the case in wavelet and Fourier analysis. Moreover, we would also like to be able to construct our orthonormal basis with some algorithm. An example of such a situation is when $G$ is a countable discrete group with a unitary representation $\nu: G \rightarrow U(\mathcal{H}), \psi \in \mathcal{H}$ is a unit vector and $\left\{\nu_{g} \psi\right\}_{g \in G}$ is an orthonormal basis for $\mathcal{H}$. However, one often requires additional properties such as smoothness, compact support or symmetry properties that are not compatible with orthonormality. If this is the case, then one is quickly led into weakening the assumption of orthonormality. One could, for instance, try to use frames. A frame in a Hilbert space $\mathcal{H}$ is a family $\left\{\psi_{i}\right\}_{i \in \mathbb{N}}$ such that there exist $A, B>0$ such that

$$
A\|\psi\|^{2} \leq \sum_{i \in \mathbb{N}}\left|\left\langle\psi, \psi_{i}\right\rangle\right|^{2} \leq B\|\psi\|^{2}
$$

for every $\psi \in \mathcal{H}$. We say that two frames, $\left\{\psi_{i}\right\}_{i \in \mathbb{N}}$ and $\left\{\tilde{\psi}_{i}\right\}_{i \in \mathbb{N}}$, are dual if $\langle\zeta, \eta\rangle=$ $\sum_{i \in \mathbb{N}}\left\langle\zeta, \psi_{i}\right\rangle\left\langle\tilde{\psi}_{i}, \eta\right\rangle$ for every $\zeta, \eta \in \mathcal{H}$. We will consider frames that are generated by a finite family $\psi_{1}, \ldots, \psi_{d}$ and a unitary representation $\nu$ of a countable discrete group $G$, i.e. such that the vectors $\left\{\nu_{g} \psi_{i}\right\}_{g \in G, 1 \leq i \leq d}$ form a frame in $\mathcal{H}$.

If we assume an additional decay property such as $\left\langle\psi_{i}, \nu_{g} \psi_{i}\right\rangle=0$ for all but finitely many $g \in G$, then it turns out that we can answer several questions about

Received by the editors June 26, 2008.

2010 Mathematics Subject Classification. Primary 42C15, 42C40, 19A13.

Key words and phrases. Frames, shift-invariant subspaces, multiresolution analysis and unitary group representations.

This research was supported in part by the Research Council of Norway, project number NFR $154077 / 420$. Some of the final work was also done with support from the project NFR 170620/V30.

(C)2010 American Mathematical Society Reverts to public domain 28 years from publication 
such frames using algebraic methods. This is the scope of the present paper. The main idea is to consider a dense and $G$-invariant subspace $\Lambda \subset \mathcal{H}$ that consists of elements with a decay property such as the one mentioned above. Now $\Lambda$ is a module over the group algebra $\mathbb{C}(G)$ in a canonical way. The properties of this module will reveal some properties of frames generated by countable discrete groups.

The idea of using modules in this way to analyze frames is not new. For instance Packer, Rieffel and others have worked on wavelets in the similar setting of projective multiresolution analysis and Hilbert modules; see [PR04, PR03, [Pac07, [DR07] and [LR07. See also Woo04, [HL00] and [FL02].

The following result is one of the main findings in this paper: Suppose that $\mathcal{H}$ is a shift-invariant subspace in $L^{2}\left(\mathbb{R}^{n}\right)$, i.e. we let $G=\mathbb{Z}^{n}$ act on $L^{2}\left(\mathbb{R}^{n}\right)$ by translations and let $\mathcal{H} \subset L^{2}\left(\mathbb{R}^{n}\right)$ be a closed $G$-invariant subspace. Suppose $\psi_{1}, \ldots, \psi_{d}$ and $\tilde{\psi}_{1}, \ldots, \tilde{\psi}_{d}$ are compactly supported and $\left\{\nu_{g} \psi_{i} \mid g \in G, 1 \leq i \leq d\right\}$ and $\left\{\nu_{g} \tilde{\psi}_{i} \mid g \in G, 1 \leq i \leq d\right\}$ form dual frames in $\mathcal{H}$. In Theorem 4.2 we prove that there must exist compactly supported $\phi_{1}, \ldots, \phi_{r}$ and $\tilde{\phi}_{1}, \ldots, \tilde{\phi}_{r}$ in $\mathcal{H}$ such that $\left\{\nu_{g} \psi_{i} \mid g \in G, 1 \leq i \leq d\right\}$ and $\left\{\nu_{g} \tilde{\psi}_{i} \mid g \in G, 1 \leq i \leq d\right\}$ form bi-orthogonal dual frames for $\mathcal{H}$, i.e. $\left\langle\nu_{g_{1}} \phi_{i}, \nu_{g_{2}} \tilde{\phi}_{j}\right\rangle=\delta_{g_{i}, g_{j}} \delta_{i, j}$. The crucial part in the proof is a theorem due to Swan, see [Swa78], that states that every finitely generated and projective module over the Laurent polynomials in $n$ variables is also a free module.

\section{Frames, modules AND UnitARY REPRESENTATIONS}

2.1. Representations of countable discrete groups. Let $G$ be a countable discrete group. For every $g \in G$, we define a corresponding unitary operator $\lambda_{g} \in$ $B\left(l^{2}(G)\right)$ with the equation

$$
\left(\lambda_{g} a\right)_{\tilde{g}}=a_{g^{-1} \tilde{g}}
$$

The operators $\left\{\lambda_{g}\right\}_{g \in G}$ form a unitary representation of $G$ onto $l^{2}(G)$. This is the left regular representation of $G$. We let $C_{r}^{*}(G)$ denote the operator norm closure of the $*$-algebra generated by the operators $\left\{\nu_{g}\right\}_{g \in G}$. This is called the reduced group $C^{*}$-algebra of $G$. More information about such algebras can be found in Dav96.

Let $f_{e}$ denote the canonical orthonormal basis element for the trivial element in $G$ and let $\tau$ denote the state on $C_{r}^{*}(G)$ such that $\tau(a)=\left\langle a f_{e}, f_{e}\right\rangle$. The map $a \mapsto\left\{a_{g}\right\}_{g \in G}$ defines an embedding $C_{r}^{*}(G) \rightarrow l^{2}(G)$ that translates Parseval's identity into $\tau\left(a a^{*}\right)=\sum_{g \in G}\left|\tau\left(a \lambda_{g}^{*}\right)\right|^{2}$ for every $a \in C_{r}^{*}(G)$. Using the polarization identity, we obtain the following useful relation, where the convergence is absolute:

$$
\tau\left(a b^{*}\right)=\sum_{g \in G} \tau\left(a \lambda_{g}^{*}\right) \tau\left(\lambda_{g} b^{*}\right) .
$$

2.2. Frames. Let $\mathcal{H}$ be a Hilbert space. We will say that a family $\left\{\zeta_{i}\right\}_{i \in \mathbb{N}} \subset \mathcal{H}$ is a frame for $\mathcal{H}$ if there exist $A, B>0$ such that

$$
A\|\zeta\|^{2} \leq \sum_{i \in \mathbb{N}}\left|\left\langle\zeta, \zeta_{i}\right\rangle\right|^{2} \leq B\|\zeta\|^{2}
$$

for every $\zeta \in \mathcal{H}$. Suppose $\left\{\tilde{\zeta}_{i}\right\}_{i \in \mathbb{N}}$ is another frame in $\mathcal{H}$. We say that this frame is a dual frame of $\left\{\zeta_{i}\right\}_{i \in \mathbb{N}}$ if

$$
\zeta=\sum_{i \in \mathbb{N}}\left\langle\zeta, \zeta_{i}\right\rangle \tilde{\zeta}_{i}=\sum_{i \in \mathbb{N}}\left\langle\zeta, \tilde{\zeta}_{i}\right\rangle \zeta_{i}
$$

for every $\zeta \in \mathcal{H}$. 
Let $\left\{e_{i}\right\}_{i \in \mathbb{N}}$ denote the canonical orthonormal basis in $l^{2}(I)$. We define the analysis operator $D: \mathcal{H} \rightarrow l^{2}(\mathbb{N})$ by the equation

$$
D \zeta=\sum_{i \in \mathbb{N}}\left\langle\zeta, \zeta_{i}\right\rangle e_{i}
$$

The analysis operator is bounded since $\|D \zeta\|^{2}=\sum_{i \in \mathbb{N}}\left|\left\langle\zeta, \zeta_{i}\right\rangle\right|^{2} \leq B\|\zeta\|^{2}$. Moreover, $D^{*} D$ is invertible since $A\|\zeta\|^{2} \leq\left\langle D^{*} D \zeta, \zeta\right\rangle$; see [Ped89, Proposition 3.2.12].

A standard result states that given a frame $\left\{\zeta_{i}\right\}_{i \in \mathbb{N}}$, then the vectors

$$
\left\{\tilde{\zeta}_{i}=\left(D^{*} D\right)^{-1} \zeta_{i}\right\}_{i \in \mathbb{N}}
$$

form a dual frame. This is proved in Dau92, Proposition 3.2.3]. We say that $\left\{\tilde{\zeta}_{i}\right\}_{i \in \mathbb{N}}$ is the canonical dual frame of $\left\{\zeta_{i}\right\}_{i \in \mathbb{N}}$. Finally, if two dual frames $\left\{\zeta_{i}\right\}_{i \in \mathbb{N}}$ and $\left\{\tilde{\zeta}_{i}\right\}_{i \in \mathbb{N}}$ satisfy $\left\langle\zeta_{i}, \tilde{\zeta}_{j}\right\rangle=\delta_{i, j}$, then we will say that they are bi-orthogonal.

2.3. Module frames. Let $\mathcal{A}$ be a unital $*$-algebra and let $E$ be a left $\mathcal{A}$-module. A Hermitian form on $E$ is a map $\langle\cdot, \cdot\rangle_{\mathcal{A}}: E \times E \rightarrow \mathcal{A}$ such that for every $a \in \mathcal{A}$ and $\zeta_{1}, \zeta_{2} \in E$, then:

(1) $\left\langle a \zeta_{1}+\zeta_{2}, \eta\right\rangle_{\mathcal{A}}=a\left\langle\zeta_{1}, \eta\right\rangle_{\mathcal{A}}+\left\langle\zeta_{2}, \eta\right\rangle_{\mathcal{A}}$

(2) $\langle\zeta, \eta\rangle_{\mathcal{A}}^{*}=\langle\eta, \zeta\rangle_{A}$.

If, moreover, $\langle\zeta, \zeta\rangle_{\mathcal{A}}=0$ implies that $\zeta=0$, then we will say that the Hermitian form is non-degenerate. The module is said to be self-dual with respect to $\langle\cdot, \cdot\rangle_{\mathcal{A}}$ if for every $\omega \in \operatorname{Hom}_{\mathcal{A}}(E, \mathcal{A})$, there exists an $\eta \in E$ such that $\omega=\langle\cdot, \eta\rangle_{\mathcal{A}}$.

Suppose $\langle\cdot, \cdot\rangle_{\mathcal{A}}$ is a non-degenerate Hermitian form on $E$. Two families $\zeta_{1}, \ldots, \zeta_{d}$ $\in E$ and $\tilde{\zeta}_{1} \ldots, \tilde{\zeta}_{d} \in E$ form dual module frames for $E$ if

$$
\langle\zeta, \eta\rangle_{\mathcal{A}}=\sum_{i=1}^{d}\left\langle\zeta, \zeta_{i}\right\rangle_{\mathcal{A}}\left\langle\tilde{\zeta}_{i}, \eta\right\rangle_{\mathcal{A}}
$$

for every $\zeta, \eta \in E$. Using the properties of $\langle\cdot, \cdot\rangle_{\mathcal{A}}$, we can easily check that $\zeta_{1}, \ldots, \zeta_{d}$ and $\tilde{\zeta}_{1} \ldots, \tilde{\zeta}_{d}$ form dual module frames if and only if

$$
\sum_{i}\left\langle\zeta, \zeta_{i}\right\rangle_{\mathcal{A}} \tilde{\zeta}_{i}=\sum_{i}\left\langle\zeta, \tilde{\zeta}_{i}\right\rangle_{\mathcal{A}} \zeta_{i}=\zeta
$$

for every $\zeta \in E$. If in addition $\left\langle\zeta_{i}, \tilde{\zeta}_{j}\right\rangle_{\mathcal{A}}=\delta_{i, j}$ for every $1 \leq i, j \leq d$, then we will say that the dual frames form dual module bases.

2.4. Projective modules. Recall that a finitely generated $\mathcal{A}$-module is said to be projective if there exists another $\mathcal{A}$-module $F$ such that $E \oplus F$ is free, i.e. $E \oplus F$ is isomorphic to $\mathcal{A}^{m}$ for an $m \in \mathbb{N}$. An $\mathcal{A}$-module is projective if and only if for every $\mathcal{A}$-module $M$ and surjective $T \in \operatorname{Hom}_{\mathcal{A}}(M, E)$ there exists an $S \in \operatorname{Hom}_{\mathcal{A}}(E, M)$ such that $S T=i d_{E}$; see [Lan02].

Lemma 2.1. A finitely generated $\mathcal{A}$-module $E$ with a non-degenerate Hermitian form has dual module frames if and only if it is projective and self-dual. Moreover, $E$ has dual module bases if and only if it is free and self-dual.

Proof. First we assume that $E$ is self-dual. Let $F$ be another $\mathcal{A}$-module with a Hermitian form and let $S \in \operatorname{Hom}_{\mathcal{A}}(E, F)$. Now, $\langle S \cdot, \eta\rangle_{\mathcal{A}} \in \operatorname{Hom}_{\mathcal{A}}(E, \mathcal{A})$ for every $\eta \in F$ and there exists an element $S^{*} \eta \in E$ such that $\langle S \zeta, \eta\rangle_{\mathcal{A}}=\left\langle\zeta, S^{*} \eta\right\rangle_{\mathcal{A}}$ for every $\zeta \in E$. We can easily check that the map $\eta \mapsto S^{*} \eta$ is $\mathcal{A}$-linear. This implies that a finitely generated module $E$ with a non-degenerate and Hermitian form is self-dual 
if and only if for every finitely generated $\mathcal{A}$-module $F$ with a Hermitian form, then every $S \in \operatorname{Hom}_{\mathcal{A}}(E, F)$ is adjointable, i.e. there exists an $S^{*} \in \operatorname{Hom}_{\mathcal{A}}(F, E)$ such that $\langle S \zeta, \eta\rangle_{\mathcal{A}}=\left\langle\zeta, S^{*} \eta\right\rangle_{\mathcal{A}}$ for every $\zeta \in E$ and $\eta \in F$.

Suppose $E$ is finitely generated, projective and self-dual. We let $\zeta_{1}, \ldots, \zeta_{n}$ be a generating set for $E$ and let $S$ denote the $\mathcal{A}$-linear map $\mathcal{A}^{d} \rightarrow E$ such that $S e_{i}=\zeta_{i}$ for $1 \leq i \leq d$. Since $E$ is projective, there exists a $T \in \operatorname{Hom}_{\mathcal{A}}\left(E, \mathcal{A}^{d}\right)$ such that $S T=1_{E}$. Now, we equip $\mathcal{A}^{d}$ with the Hermitian form $\langle a, b\rangle_{\mathcal{A}}=\sum_{i=1}^{d} a_{i} b_{i}^{*}$. The map $T$ is adjointable since $E$ is self-dual. Let $\tilde{\zeta}_{i}=T^{*} e_{i}$ for $1 \leq i \leq d$. We see that whenever $\zeta \in E$, then

$$
\sum_{i=1}^{d}\left\langle\zeta, \tilde{\zeta}_{i}\right\rangle_{\mathcal{A}} \zeta_{i}=\sum_{i=1}^{d}\left\langle\zeta, T^{*} \zeta_{i}\right\rangle_{\mathcal{A}} S e_{i}=S \sum_{i=1}^{d}\left\langle T \zeta, \zeta_{i}\right\rangle_{\mathcal{A}} e_{i}=S T \zeta=\zeta
$$

so $\zeta_{1}, \ldots, \zeta_{d}$ and $\tilde{\zeta}_{1} \ldots, \tilde{\zeta}_{d}$ form dual module frames for $E$. Suppose, moreover, that $E$ is free and assume that $\zeta_{1}, \ldots, \zeta_{d}$ form an $\mathcal{A}$-basis for $E$. Now $S$ is invertible, so if we define $\tilde{\zeta}_{i}=\left(S^{-1}\right)^{*} e_{i}$, then we see that

$$
\left\langle\zeta_{i}, \tilde{\zeta}_{j}\right\rangle_{\mathcal{A}}=\left\langle\zeta_{i},\left(S^{-1}\right)^{*} e_{i}\right\rangle_{\mathcal{A}}=\left\langle S^{-1} \zeta_{i}, e_{j}\right\rangle_{\mathcal{A}}=\left\langle e_{i}, e_{j}\right\rangle_{\mathcal{A}}=\delta_{i, j}
$$

For the converse statement, suppose $\zeta_{1}, \ldots, \zeta_{d}$ and $\tilde{\zeta}_{1} \ldots, \tilde{\zeta}_{d}$ form dual module frames for $E$ and let $\omega \in \operatorname{Hom}_{\mathcal{A}}(E, \mathcal{A})$. If $\zeta \in E$, then

$$
\omega(\zeta)=\omega\left(\sum_{i=1}^{d}\left\langle\zeta, \tilde{\zeta}_{i}\right\rangle_{\mathcal{A}} \zeta_{i}\right)=\left\langle\zeta, \sum_{i=1}^{d} \omega\left(\zeta_{i}\right)^{*} \tilde{\zeta}_{i}\right\rangle_{\mathcal{A}},
$$

so $E$ is self-dual. Now, we define $S, \tilde{S} \in \operatorname{Hom}_{\mathcal{A}}\left(\mathcal{A}^{d}, E\right)$ such that $S e_{i}=\zeta_{i}$ and $\tilde{S} e_{i}=\tilde{\zeta}_{i}$ for $1 \leq i \leq d$. A short computation shows that $S$ and $\tilde{S}$ are adjointable and

$$
S^{*} \zeta=\sum_{i=1}^{d}\left\langle\zeta, e_{i}\right\rangle_{\mathcal{A}} \zeta_{i}, \quad \quad \tilde{S}^{*} \zeta=\sum_{i=1}^{d}\left\langle\zeta, e_{i}\right\rangle_{\mathcal{A}} \tilde{\zeta}_{i} .
$$

Moreover, we can easily check that $S \tilde{S}^{*}=\tilde{S} S^{*}=1_{E}$. This implies that

$$
\zeta \mapsto S \zeta \oplus\left(1-\tilde{S}^{*} S\right) \zeta
$$

defines an $\mathcal{A}$-linear isomorphism from $\mathcal{A}^{d}$ to $E \oplus \operatorname{ker} \tilde{S}^{*} S$, so $E$ is projective.

\section{The Module of COMPaCtLy SUPported VeCtors in $\mathcal{H}$}

We let $\mathcal{A}$ denote the $*$-algebra of finite linear combinations of elements in $\left\{\lambda_{g}\right\}_{g \in G}$ $\subset C_{r}^{*}(G)$. Moreover, let $\mathcal{H}$ be a Hilbert space with a unitary representation $\nu: G \rightarrow$ $U(\mathcal{H})$.

The action $\nu$ defines a $*$-homomorphism $\pi_{0}: \mathcal{A} \rightarrow B(\mathcal{H})$ such that $\pi_{0}\left(\lambda_{g}\right)=\nu_{g}$. However, we cannot necessarily extend this to a $*$-homomorphism $C_{r}^{*}(G) \rightarrow B(\mathcal{H})$, unless $G$ is amenable, in which case it is well known that $C_{r}(G)$ coincides with the full group $C^{*}$-algebra on $G$. We will occasionally apply the operator norm inherited from $C_{r}^{*}(G)$ on elements in $\mathcal{A}$. This norm will be denoted by $\|\cdot\|$. Moreover, the restriction of the cone of positive elements in $C_{r}^{*}(G)$ defines a cone of positive elements in $\mathcal{A}$.

We let $\Lambda \subset \mathcal{H}$ be a subspace such that:

(1) $\pi_{0}(a) \Lambda \subset \Lambda$ for every $a \in \mathcal{A}$,

(2) if $\zeta, \eta \in \Lambda$, then $\left\langle\zeta, \nu_{g} \eta\right\rangle=0$ for all but finitely many $g \in G$. 
The first condition means that $\Lambda$ is an $\mathcal{A}$-module when equipped with the product $a, \zeta \mapsto \pi_{0}(a) \zeta$. The motivating example for this occurs when $\Lambda$ is a subspace of $L^{2}\left(\mathbb{R}^{n}\right)$ that is formed by functions of compact support and $G=\mathbb{Z}^{n} \subset \mathbb{R}^{n}$ acts on $L^{2}\left(\mathbb{R}^{n}\right)$ by translations. Note that most computations and definitions work out nicely if we weaken the decay assumption, i.e. instead of assuming (2), we assume that $\left\{\left\langle\zeta, \nu_{g} \eta\right\rangle\right\}_{g \in G} \in l^{1}(G)$.

Now, we define a Hermitian form $\langle\cdot, \cdot\rangle_{\mathcal{A}}$ on the $\mathcal{A}$-module $\Lambda$ with the following equation:

$$
\langle\zeta, \eta\rangle_{\mathcal{A}}=\sum_{g \in G}\left\langle\zeta, \nu_{g} \eta\right\rangle \lambda_{g}
$$

We list some of its properties in the following proposition:

Proposition 3.1. Let $\zeta_{1}, \zeta_{2}, \zeta, \eta \in \Lambda$ and $a \in \mathcal{A}$. The map $\langle\cdot, \cdot\rangle_{\mathcal{A}}$ satisfies the following properties:

(1) $\langle\zeta, \zeta\rangle_{\mathcal{A}} \geq 0$ and $\langle\zeta, \zeta\rangle_{\mathcal{A}}=0$ if and only if $\zeta=0$.

(2) $\left\langle a \zeta_{1}+\zeta_{2}, \eta\right\rangle_{\mathcal{A}}=a\left\langle\zeta_{1}, \eta\right\rangle_{\mathcal{A}}+\left\langle\zeta_{2}, \eta\right\rangle_{A}$.

(3) $\langle\zeta, \eta\rangle_{\mathcal{A}}^{*}=\langle\eta, \zeta\rangle_{A}$.

(4) $\langle\zeta, \eta\rangle_{A}\langle\eta, \zeta\rangle_{\mathcal{A}} \leq\left\|\langle\zeta, \zeta\rangle_{\mathcal{A}}\right\|\langle\eta, \eta\rangle_{\mathcal{A}}$.

Proof. We let $a, b \in l^{2}(G)$ and compute with respect to the standard inner product on $l^{2}(G)$ :

$$
\begin{aligned}
\left\langle\langle\zeta, \eta\rangle_{\mathcal{A}} a, b\right\rangle & =\sum_{g \in G} \sum_{f \in G}\left\langle\zeta, \nu_{f} \eta\right\rangle a_{f^{-1} g} \bar{a}_{g}=\sum_{g \in G} \sum_{f \in G}\left\langle\zeta, \nu_{f-1} \eta\right\rangle a_{g} \bar{a}_{f^{-1}} g \\
& =\left\langle a,\langle\eta, \zeta\rangle_{\mathcal{A}} b\right\rangle .
\end{aligned}
$$

This shows that $\langle\zeta, \eta\rangle_{\mathcal{A}}^{*}=\langle\eta, \zeta\rangle_{\mathcal{A}}$ for every $\zeta, \eta \in \Lambda$.

To see that $\langle\zeta, \zeta\rangle_{\mathcal{A}} \geq 0$, we pick $a \in l^{2}(G)$ with finite support and consider the following computation:

$$
\begin{aligned}
\left\langle\langle\zeta, \zeta\rangle_{\mathcal{A}} a, a\right\rangle & =\sum_{g \in G} \sum_{f \in G}\left\langle\zeta, \nu_{f} \zeta\right\rangle a_{f^{-1} g} \bar{a}_{g}=\sum_{f \in G} \sum_{g \in G}\left\langle\zeta, \nu_{g^{-1}} \zeta\right\rangle a_{g} \bar{a}_{f} \\
& =\left\langle\sum_{g \in G} a_{g} \nu_{g} \zeta, \sum_{f \in G} a_{f} \nu_{f} \zeta\right\rangle \geq 0 .
\end{aligned}
$$

Now, let $a \in l^{2}(G)$ be arbitrary and let $\left\{a_{n}\right\}_{n \in \mathbb{N}} \subset l^{2}(G)$ be a sequence of elements with finite supports such that $\lim _{n}\left\|a_{n}-a\right\|=0$. We see that $\left\langle\langle\zeta, \zeta\rangle_{\mathcal{A}} a, a\right\rangle=$ $\lim _{n}\left\langle\langle\zeta, \zeta\rangle_{\mathcal{A}} a_{n}, a_{n}\right\rangle \geq 0$, so $\langle\zeta, \zeta\rangle_{\mathcal{A}} \geq 0$ for every $\zeta \in \Lambda$. Moreover, if $\langle\zeta, \zeta\rangle_{\mathcal{A}}=0$, then $\left\langle\zeta, \nu_{g} \zeta\right\rangle=0$ for every $g \in G$ since $\left\{\lambda_{g}\right\}_{g}$ yields an orthonormal basis for $l^{2}(G)$.

Whenever $a \in \mathcal{A}$, we have the following relation:

$$
\langle a \zeta, \eta\rangle_{\mathcal{A}}=\sum_{g \in G} \sum_{f \in G} a_{f}\left\langle\nu_{g} \zeta, \nu_{f} \eta\right\rangle \lambda_{f}=\sum_{g \in G} \sum_{f \in G} a_{g} \lambda_{g}\left\langle\zeta, \nu_{f} \eta\right\rangle \lambda_{f}=a\langle\zeta, \eta\rangle_{\mathcal{A}}
$$

Now, it's easy to see that $\left\langle a \zeta_{1}+\zeta_{2}, \eta\right\rangle_{\mathcal{A}}=a\left\langle\zeta_{1}, \eta\right\rangle_{\mathcal{A}}+\left\langle\zeta_{2}, \eta\right\rangle_{\mathcal{A}}$ whenever $\zeta_{1}, \zeta_{2}, \eta \in \Lambda$.

Finally, we will prove the Cauchy-Schwarz-like inequality (44) as follows:

$$
\begin{aligned}
0 & \leq\left\langle\langle\zeta, \eta\rangle_{\mathcal{A}}\left\|\langle\eta, \eta\rangle_{\mathcal{A}}\right\|^{-1} \eta-\zeta,\langle\zeta, \eta\rangle_{\mathcal{A}}\left\|\langle\eta, \eta\rangle_{\mathcal{A}}\right\|^{-1} \eta-\zeta\right\rangle_{\mathcal{A}} \\
& =\langle\zeta, \eta\rangle_{\mathcal{A}}\langle\eta, \eta\rangle_{\mathcal{A}}\langle\eta, \zeta\rangle_{\mathcal{A}}\left\|\langle\eta, \eta\rangle_{\mathcal{A}}\right\|^{-2}-2\langle\zeta, \eta\rangle_{\mathcal{A}}\langle\eta, \zeta\rangle_{\mathcal{A}}\left\|\langle\eta, \eta\rangle_{\mathcal{A}}\right\|^{-1}+\langle\zeta, \zeta\rangle_{\mathcal{A}} .
\end{aligned}
$$


If $a, b$ are elements in a $C^{*}$-algebra and $b$ is positive, then $a^{*} b a \leq\|b\| a^{*} a$; see BR87, 2.2.2]. Since $\langle\zeta, \zeta\rangle_{\mathcal{A}}$ is positive in $C_{r}^{*}(G)$, we obtain the inequality

$$
\langle\zeta, \eta\rangle_{\mathcal{A}}\langle\eta, \eta\rangle_{\mathcal{A}}\langle\eta, \zeta\rangle_{\mathcal{A}} \leq\left\|\langle\eta, \eta\rangle_{\mathcal{A}}\right\|^{-1}\langle\zeta, \eta\rangle_{\mathcal{A}}\langle\eta, \zeta\rangle_{\mathcal{A}} .
$$

Using this inequality, we obtain the following:

$$
0 \leq-\langle\zeta, \eta\rangle_{\mathcal{A}}\langle\eta, \zeta\rangle_{\mathcal{A}}\left\|\langle\eta, \eta\rangle_{\mathcal{A}}\right\|^{-1}+\langle\zeta, \zeta\rangle_{\mathcal{A}} .
$$

We see immediately that this is equivalent to (4).

Remark 3.2. The module $\Lambda$ is closely related to a Hilbert module introduced by Packer and Rieffel in PR04. They considered the space

$$
\Xi=\left\{f \in C_{b}\left(\mathbb{R}^{n}\right) \mid \sum_{g \in \mathbb{Z}^{n}} f(\cdot-g) \bar{f}(\cdot-g) \text { converges uniformly }\right\} .
$$

This space $\Xi$ is a $C\left(\mathbb{T}^{n}\right)$-module in a canonical way. It is also a $C\left(\mathbb{T}^{n}\right)$-Hilbert module, see [Lan95, when equipped with the $C\left(\mathbb{T}^{n}\right)$-valued Hermitian form

$$
\left\langle f_{1}, f_{2}\right\rangle^{\prime}=\sum_{g \in \mathbb{Z}^{n}} f_{1}(\cdot-g) \bar{f}_{2}(\cdot-g) .
$$

They introduced this Hilbert module in order to define projective multiresolution analysis; see also [DR07] and [LR07. We will not deal with projective resolution analysis, but only make a few comments on the relation between $\Lambda$ and the Hilbert module $\Xi$.

Suppose, as in the previous section, that $G=\mathbb{Z}^{n}$ and assume that the action $\nu$ on $L^{2}\left(\mathbb{R}^{n}\right)$ is given as follows: $\left(\nu_{g} \zeta\right)(x)=\zeta(x-g), g \in G$. We let $\Lambda$ denote the set of compactly supported functions in $L^{2}\left(\mathbb{R}^{n}\right)$. The Fourier transform relates the $\mathcal{A}$-module $\Lambda$ to the Hilbert module $\Xi$ in the following way: First, we note that if $\zeta \in \Lambda \subset L^{1}\left(\mathbb{R}^{n}\right)$, then $\widehat{\zeta}$ is continuous. Let $G^{\perp}=\left\{y \in \mathbb{R}^{n} \mid\langle x, y\rangle \in \mathbb{Z}\right.$ for every $x \in$ $G\}$ and let $X=\mathbb{R}^{n} / G^{\perp}$. Moreover, let $\mu$ be the Haar measure on $X \simeq \mathbb{T}^{n}$ with the normalization such that $\int_{\mathbb{R}^{n}} f d x=\int_{X} \sum_{g \in G^{\perp}} f(x-g) d \mu([x])$ for every $f \in C_{c}\left(\mathbb{R}^{n}\right)$. Now if $\zeta_{1}, \zeta_{2}, \eta \in \Lambda$, then

$$
\begin{aligned}
\left(\left\langle\widehat{\left.\zeta_{1}, \zeta_{2}\right\rangle_{\mathcal{A}}} \eta\right)(\sigma)\right. & =\sum_{g \in G}\left\langle\zeta_{1}, \nu_{g} \zeta_{2}\right\rangle \widehat{\nu_{g} \eta}(\sigma)=\sum_{g \in G} \int_{\mathbb{R}^{n}} \hat{\zeta}_{1}(y) \overline{\hat{\zeta}_{2}}(y) \overline{(g, y)} d y(g, \sigma) \hat{\eta}(\sigma) \\
& =\left(\mathcal{F}^{-1} \mathcal{F}\left\langle\zeta_{1}, \zeta_{2}\right\rangle^{\prime}\right)(\sigma) \hat{\eta}(\sigma)=\left\langle\hat{\zeta}_{1}, \hat{\zeta}_{2}\right\rangle^{\prime}(\sigma) \hat{\eta}(\sigma) .
\end{aligned}
$$

This means that the Fourier transform takes $\Lambda$ to a subspace in $\Xi$ and takes the Hermitian form $\langle\cdot, \cdot\rangle_{\mathcal{A}}$ to $\langle\cdot, \cdot\rangle^{\prime}$.

Remark 3.3. A countable group $G$ is called amenable if there exists a left translationinvariant state on $l^{\infty}(G)$. Finite groups and Abelian groups are examples of amenable groups. This is a standard result that is proved in [Dav96, VII.2]. Moreover, if $G$ is an amenable discrete group, $\nu$ is a unitary representation of $G$ on $\mathcal{H}$ and $\mathcal{B}$ is the $C^{*}$-algebra generated by the representation $\nu$, then there exists a *-homomorphism $\pi: C_{r}^{*}(G) \rightarrow \mathcal{B}$ such that $\pi\left(\lambda_{g}\right)=\nu_{g}$ for every $g \in G$. This result is also standard and can be found in [Dav96, Theorem V.II.2.8]. We note that if such a $\pi$ exists, then it is unique. This follows from extension by continuity since $\mathcal{A}$ is a dense $*$-subalgebra in $C_{r}^{*}(G)$.

If $G$ is amenable, then we can always complete $\Lambda$ into a $C_{r}^{*}(G)$-Hilbert module $\Lambda_{0} \subset \mathcal{H}$. Let $\tilde{\Lambda}$ denote the vector space $\pi\left(C_{r}^{*}(G)\right) \Lambda$. The space $\tilde{\Lambda}$ has a canonical $C_{r}^{*}(G)$-module structure, and the Hermitian form $\langle\cdot, \cdot\rangle_{\mathcal{A}}$ extends uniquely to a 
$C_{r}^{*}(G)$-valued Hermitian form $\langle\cdot, \cdot\rangle_{\mathcal{A}}$ on $\tilde{\Lambda}$. The map $\zeta \mapsto\left\|\langle\zeta, \zeta\rangle_{C_{r}^{*}(G)}\right\|_{\text {op }}^{1 / 2}$ defines a norm on $\tilde{\Lambda}$, so the usual completion procedure of a normed vector space defines a left module over $C_{r}^{*}(G)$. If a sequence $\left\{\zeta_{n}\right\}_{n} \subset \tilde{\Lambda}$ is a Cauchy sequence with respect to this norm, then it is also a Cauchy sequence with respect to the norm on $\mathcal{H}$. Since $\mathcal{H}$ is complete, we see that the completion of $\tilde{\Lambda}$ is contained in $\mathcal{H}$. We let $\Lambda_{0} \subset \mathcal{H}$ denote the subspace formed by this completion. The Hermitian form $\langle\cdot, \cdot\rangle_{\mathcal{A}}$ now extends to a non-degenerate $C_{r}^{*}(G)$-valued Hermitian form $\langle\cdot, \cdot\rangle_{C_{r}^{*}(G)}: \Lambda_{0} \times \Lambda_{0} \rightarrow$ $C_{r}^{*}(G)$ that makes $\Lambda_{0}$ into a Hilbert module, i.e. $\langle\zeta, \zeta\rangle_{C_{r}^{*}(G)} \geq 0$ for every $\zeta \in \Lambda_{0}$ and $\langle\zeta, \eta\rangle_{C_{r}^{*}(G)}\langle\eta, \zeta\rangle_{C_{r}^{*}(G)} \leq\left\|\langle\zeta, \zeta\rangle_{C_{r}^{*}(G)}\right\|\langle\eta, \eta\rangle_{C_{r}^{*}(G)}$ for every $\zeta, \eta \in \Lambda_{0}$.

3.1. $G$-frames. We will say that a finite family of vectors $\zeta_{1}, \ldots, \zeta_{d} \in \mathcal{H}$ form a $G$ frame in $\mathcal{H}$ if the vectors $\left\{\nu_{g} \zeta_{i} \mid g \in G, 1 \leq i \leq d\right\}$ form a frame in $\mathcal{H}$. The following theorem says that we can tell if two families, $\left\{\zeta_{i}\right\}_{1 \leq i \leq d}$ and $\left\{\tilde{\zeta}_{i}\right\}_{1 \leq i \leq d}$, form dual $G$-frames for a closed subspace in $\mathcal{H}$ by looking at a corresponding submodule in $\Lambda$. A similar result can be found in [PR04].

Theorem 3.4. Suppose that $E \subset \Lambda$ is an $\mathcal{A}$-submodule and let $\zeta_{1}, \ldots, \zeta_{d}, \tilde{\zeta}_{1} \ldots, \tilde{\zeta}_{d}$ $\in E$. The following conditions are equivalent:

(1) The families $\left\{\zeta_{i}\right\}_{1 \leq i \leq d}$ and $\left\{\tilde{\zeta}_{i}\right\}_{1 \leq i \leq d}$ form dual $G$-frames for $\bar{E}$.

(2) The families $\left\{\zeta_{i}\right\}_{1 \leq i \leq d}$ and $\left\{\tilde{\zeta}_{i}\right\}_{1 \leq i \leq d}$ form dual module frames for $E$.

Proof. Suppose $\zeta \in E$ and that the families $\left\{\zeta_{i}\right\}_{1 \leq i \leq d}$ and $\left\{\zeta_{i}\right\}_{1 \leq i \leq d}$ form dual $G$-frames for the closure of $E$ in $\mathcal{H}$. We see that

$$
\zeta=\sum_{g, i}\left\langle\zeta, \nu_{g} \zeta_{i}\right\rangle \nu_{g} \tilde{\zeta}_{i}=\sum_{i}\left\langle\zeta, \zeta_{i}\right\rangle_{\mathcal{A}} \tilde{\zeta}_{i},
$$

so $\left\{\zeta_{i}\right\}_{1 \leq i \leq d}$ and $\left\{\zeta_{i}\right\}_{1 \leq i \leq d}$ form dual module frames for $E$.

If $\zeta, \eta \in \Lambda$, then

$$
\begin{aligned}
\sum_{g \in G}\left\langle\zeta, \nu_{g} \eta\right\rangle\left\langle\nu_{g} \eta, \zeta\right\rangle & =\sum_{g \in G} \tau\left(\langle\zeta, \eta\rangle_{\mathcal{A}} \lambda_{g}^{*}\right) \tau\left(\lambda_{g}\langle\eta, \zeta\rangle_{\mathcal{A}}\right)=\tau\left(\langle\zeta, \eta\rangle_{\mathcal{A}}\langle\eta, \zeta\rangle_{\mathcal{A}}\right) \\
& \leq \tau\left(\langle\zeta, \zeta\rangle_{\mathcal{A}}\right)\left\|\langle\eta, \eta\rangle_{\mathcal{A}}\right\|=\|\zeta\|^{2}\left\|\langle\eta, \eta\rangle_{\mathcal{A}}\right\| .
\end{aligned}
$$

This implies that $\left\{\left\langle\zeta, \nu_{g} \eta\right\rangle\right\}_{g \in G}$ is contained in $l^{2}(G)$. Moreover, if $\left\{\zeta_{n}\right\}_{n}$ is a sequence in $\mathcal{H}$ that converges to $\zeta \in \mathcal{H}$, then $l^{2}-\lim _{n}\left\{\left\langle\zeta_{n}, \nu_{g} \eta\right\rangle\right\}_{g \in G}=\left\{\left\langle\zeta, \nu_{g} \eta\right\rangle\right\}_{g \in G}$.

Suppose that $\left\{\zeta_{i}\right\}_{1 \leq i \leq d}$ and $\left\{\tilde{\zeta}_{i}\right\}_{1 \leq i \leq d}$ form dual module frames for $E$. If $\zeta, \eta$ are contained in the closure of $E$, let $\left\{\zeta^{(n)}\right\}_{n \in \mathbb{N}}$ and $\left\{\eta^{(n)}\right\}_{n \in \mathbb{N}}$ be sequences in $E$ that converge to $\zeta$ and $\eta$. We see that

$$
\begin{aligned}
\langle\zeta, \eta\rangle & =\lim _{n}\left\langle\zeta^{(n)}, \eta^{(n)}\right\rangle=\lim _{n} \tau\left(\left\langle\zeta^{(n)}, \eta^{(n)}\right\rangle_{\mathcal{A}}\right)=\lim _{n} \tau\left(\sum_{i}\left\langle\zeta^{(n)}, \zeta_{i}\right\rangle_{\mathcal{A}}\left\langle\tilde{\zeta}_{i}, \eta^{(n)}\right\rangle_{\mathcal{A}}\right) \\
& =\lim _{n} \sum_{i, g} \tau\left(\left\langle\zeta^{(n)}, \zeta_{i}\right\rangle_{\mathcal{A}} \lambda_{g}^{*}\right) \tau\left(\lambda_{g}\left\langle\tilde{\zeta}_{i}, \eta^{(n)}\right\rangle_{\mathcal{A}}\right)=\lim _{n} \sum_{g, i}\left\langle\zeta^{(n)}, \nu_{g} \zeta_{i}\right\rangle\left\langle\nu_{g} \tilde{\zeta}_{i}, \eta^{(n)}\right\rangle \\
& =\sum_{g, i}\left\langle\zeta, \nu_{g} \zeta_{i}\right\rangle\left\langle\nu_{g} \tilde{\zeta}_{i}, \eta\right\rangle .
\end{aligned}
$$

To prove that $\zeta_{1}, \ldots, \zeta_{d}$ form a $G$-frame for the closure of $E$ in $\mathcal{H}$ we must show that there exist $A, B>0$ such that

$$
A\|\zeta\|^{2} \leq \sum_{g, i}\left|\left\langle\zeta, \nu_{g} \zeta_{i}\right\rangle\right|^{2} \leq B\|\zeta\|^{2}
$$


for every $\zeta$ in the closure of $E$. The second inequality of (3.4) is satisfied if we set $B=\sum_{i}\left\|\left\langle\zeta_{i}, \zeta_{i}\right\rangle_{\mathcal{A}}\right\|$. Moreover, we have that

$$
\begin{aligned}
\|\zeta\|^{2} & =\sup _{\|\eta\| \leq 1}|\langle\zeta, \eta\rangle|^{2}=\sup _{\|\eta\| \leq 1}\left|\sum_{g, i}\left\langle\zeta, \nu_{g} \zeta_{i}\right\rangle\left\langle\nu_{g} \tilde{\zeta}_{i}, \eta\right\rangle\right|^{2} \\
& \left.\leq \sup _{\|\eta\| \leq 1} \sum_{g, i}\left|\left\langle\zeta, \nu_{g} \zeta_{i}\right\rangle\right|^{2} \sum_{g, i}\left|\left\langle\eta, \nu_{g} \tilde{\zeta}_{i}\right\rangle\right|^{2} \leq \sup _{\|\eta\| \leq 1} \sum_{g, i}\left|\left\langle\zeta, \nu_{g} \zeta_{i}\right\rangle\right|^{2}\|\eta\|^{2} \sum_{i} \| \tilde{\langle}_{i}, \tilde{\zeta}_{i}\right\rangle_{\mathcal{A}} \| \\
& \leq \sum_{g, i}\left|\left\langle\zeta, \nu_{g} \zeta_{i}\right\rangle\right|^{2} \sum_{i}\left\|\left\langle\tilde{\zeta}_{i}, \tilde{\zeta}_{i}\right\rangle_{\mathcal{A}}\right\| .
\end{aligned}
$$

So, if we let $A=\left(\sum_{i}\left\|\left\langle\tilde{\zeta}_{i}, \tilde{\zeta}_{i}\right\rangle_{\mathcal{A}}\right\|\right)^{-1}$, then $A\|\zeta\|^{2} \leq \sum_{g, i}\left|\left\langle\zeta, \nu_{g} \zeta_{i}\right\rangle\right|^{2}$ for every $\zeta$ in the closure of $E$ in $\mathcal{H}$. Finally, we note that this argument can also be done for the family $\tilde{\zeta}_{1}, \ldots, \tilde{\zeta}_{d}$, so the families $\left\{\zeta_{i}\right\}_{1 \leq i \leq d}$ and $\left\{\zeta_{i}\right\}_{1 \leq i \leq d}$ form dual $G$-frames for the closure of $E$ in $\mathcal{H}$.

Corollary 3.5. Suppose that $E \subset \Lambda$ is an $\mathcal{A}$-submodule and let $\zeta_{1}, \ldots, \zeta_{d}, \tilde{\zeta}_{1} \ldots, \tilde{\zeta}_{d}$ $\in E$. The following conditions are equivalent:

(1) The families $\left\{\zeta_{i}\right\}_{1 \leq i \leq d}$ and $\left\{\zeta_{i}\right\}_{1 \leq i \leq d}$ form bi-orthogonal $G$-frames for $\bar{E}$.

(2) The families $\left\{\zeta_{i}\right\}_{1 \leq i \leq d}$ and $\left\{\zeta_{i}\right\}_{1 \leq i \leq d}$ form dual module bases for $E$.

Proof. This follows directly from the previous theorem and the definition of $\langle\cdot, \cdot\rangle_{\mathcal{A}}$.

\section{Shift-InVariant SUbSpaces in $L^{2}\left(\mathbb{R}^{n}\right)$}

In this section we will apply our results to shift-invariant subspaces in $L^{2}\left(\mathbb{R}^{n}\right)$. We let $G=\mathbb{Z}^{n}$ and consider the representation $\nu: G \rightarrow \mathcal{U}\left(L^{2}\left(\mathbb{R}^{n}\right)\right)$ such that

$$
\left(\nu_{g} \zeta\right)(x)=\zeta(x-g), g \in G .
$$

A closed subspace $\mathcal{V} \subset L^{2}\left(\mathbb{R}^{n}\right)$ is said to be shift-invariant if $\nu_{g} \mathcal{V} \subset \mathcal{V}$ for every $g \in G$. Note that since $G=\mathbb{Z}^{n}$, the algebra $\mathcal{A}$ is isomorphic to the $*$-algebra of Laurent polynomials in $n$ variables.

We let $\Lambda$ denote the set of functions in $L^{2}\left(\mathbb{R}^{n}\right)$ of compact support. If $\zeta_{1}, \ldots, \zeta_{d} \in$ $\Lambda$ form a $G$-frame for a closed subspace $\mathcal{V} \subset L^{2}\left(\mathbb{R}^{n}\right)$, then we will see that the structure of $\mathcal{V}$ is closely related to the structure of the $\mathcal{A}$-module $\mathcal{A} \zeta_{1}+\cdots+\mathcal{A} \zeta_{d}$.

A frame $\left\{\zeta_{i}\right\}_{i \in \mathbb{N}}$ in a Hilbert space $\mathcal{H}$ is said to be a Parseval frame for $\mathcal{H}$ if

$$
\langle\zeta, \eta\rangle=\sum_{i \in \mathbb{N}}\left\langle\zeta, \zeta_{i}\right\rangle\left\langle\zeta_{i}, \eta\right\rangle
$$

for every $\zeta, \eta \in \mathcal{H}$.

Theorem 4.1. If $\left\{\nu_{g} \zeta\right\}_{g \in G} \subset \mathcal{V} \subset L^{2}\left(\mathbb{R}^{n}\right)$ is a Parseval frame for $\mathcal{V}$ and $\zeta$ has compact support, then $\left\{\nu_{g} \zeta\right\}_{g \in G}$ is an orthonormal basis for $\mathcal{V}$.

Proof. Let $E$ denote the $\mathcal{A}$-module that consists of the compactly supported elements in $\mathcal{V}$. By Theorem 3.4, $\zeta$ forms a module frame for $E$ such that

$$
\left\langle\eta_{1}, \eta_{2}\right\rangle_{\mathcal{A}}=\left\langle\eta_{1}, \zeta\right\rangle_{\mathcal{A}}\left\langle\zeta, \eta_{2}\right\rangle_{\mathcal{A}}
$$


for every $\eta_{1}, \eta_{2} \in E$. This implies that $\langle\zeta, \zeta\rangle_{\mathcal{A}}^{2}=\langle\zeta, \zeta\rangle_{\mathcal{A}}$. Moreover, $\langle\zeta, \zeta\rangle_{\mathcal{A}}$ is selfadjoint and non-zero. The Fourier transform yields a $*$-isomorphism between $C_{r}^{*}\left(\mathbb{Z}^{n}\right)$ and $C\left(\mathbb{T}^{n}\right)$; see [Dav96, Proposition VII.1.1]. The only selfadjoint idempotents in this algebra are 1 and 0 , so $\langle\zeta, \zeta\rangle_{\mathcal{A}}=1$. If we apply Corollary 3.5, we see that $\left\langle\zeta, \nu_{g} \zeta\right\rangle=\delta_{g, 0}$.

A fundamental fact about polynomial rings in $n$ variables over a field is that every finitely generated and projective module over such a ring is free. This is known as Serre's conjecture and was an open problem for several years until it was proved independently by Quillen and Suslin in 1976. Richard G. Swan proved the analogous result for Laurent Polynomials in $n$ variables in [Swa78; see also Lam78. To us this means that every finitely generated and projective module over $\mathcal{A}$ is free. Later this was generalized to an even larger class of rings by Gubeladze in Gub88; see also Lam06. However, we shall now see that the theorem by Swan has consequences for the structure of finitely generated shift-invariant subspaces in $L^{2}\left(\mathbb{R}^{n}\right)$.

Theorem 4.2. If $\zeta_{1}, \ldots, \zeta_{d}$ and $\tilde{\zeta}_{1}, \ldots, \tilde{\zeta}_{d}$ ore compactly supported and form dual $G$-frames for a closed subspace $\mathcal{K} \subset L^{2}\left(\mathbb{R}^{n}\right)$, then there exist bi-orthogonal $G$-frames $\eta_{1}, \ldots, \eta_{r}$ and $\tilde{\eta}_{1}, \ldots, \tilde{\eta}_{d}$ with compact supports in $\mathcal{K}$.

Proof. Let $E \subset \Lambda$ denote the $\mathcal{A}$-module generated by $\zeta_{1}, \ldots, \zeta_{r}$. By Theorem 3.4. $E$ is self-dual and projective. However, since $E$ is a finitely generated projective module over $\mathcal{A}$, it is necessarily free. If we apply Lemma 2.1, we see that since $E$ is self-dual, there exist $\eta_{1}, \ldots, \eta_{r}$ and $\tilde{\eta}_{1}, \ldots, \tilde{\eta}_{r}$ in $E$ such that $\left\langle\eta_{i}, \tilde{\eta}_{j}\right\rangle_{\mathcal{A}}=\delta_{i, j}$. If we apply Corollary 3.5, we see that $\eta_{1}, \ldots, \eta_{r}$ and $\tilde{\eta}_{1}, \ldots, \tilde{\eta}_{r}$ form bi-orthogonal $G$-frames for $\mathcal{K}$.

\section{Two APPLICATIONS TO MULTIRESOLUTION ANALYSIS}

Let $G=\mathbb{Z}^{n}$, let $\nu$ denote the group action defined in the previous section and let $\Lambda$ denote a set of compactly supported elements in $L^{2}\left(\mathbb{R}^{n}\right)$. Moreover, let $A \in G L_{n}(\mathbb{R})$ such that $A G \subset G$. We assume that every eigenvalue of $A$ has absolute value strictly greater than 1 . The determinant of $A$ is an integer. Let $q=|\operatorname{det} A|$. We define a unitary operator on $L^{2}\left(\mathbb{R}^{n}\right)$ with the equation $U \zeta(t)=\sqrt{q} \zeta(A t)$.

Definition 5.1. We will say that $U$ and $\phi_{1}, \ldots, \phi_{d}, \tilde{\phi}_{1}, \ldots, \tilde{\phi}_{d} \in L^{2}\left(\mathbb{R}^{n}\right)$ generate a multiresolution analysis, MRA for short, if the following properties are satisfied:

(1) The families $\phi_{1}, \ldots, \phi_{d}$ and $\tilde{\phi}_{1}, \ldots, \tilde{\phi}_{d}$ form dual $G$-frames for a closed subspace $\mathcal{V} \subset L^{2}\left(\mathbb{R}^{n}\right)$.

(2) The subspace $U \mathcal{V}$ contains $\mathcal{V}$.

(3) The union $\bigcup_{k \in \mathbb{Z}} U^{k} \mathcal{V}$ is dense in $L^{2}\left(\mathbb{R}^{n}\right)$.

(4) The intersection $\bigcap_{k \in \mathbb{Z}} U^{k} \mathcal{V}$ equals the trivial subspace $\{0\}$.

The subspace $\mathcal{V}$ is called the scaling space and the subspace $U \mathcal{V} \ominus \mathcal{V}$ is called the wavelet space. The families $\phi_{1}, \ldots, \phi_{d}$ and $\tilde{\phi}_{1}, \ldots, \tilde{\phi}_{d}$ are said to be dual scaling families for the MRA. Two families $\psi_{1}, \ldots, \psi_{r} \in U \mathcal{V}$ and $\tilde{\psi}_{1}, \ldots, \tilde{\psi}_{r} \in U \mathcal{V}$ are said to be dual MRA wavelet families if they form dual $G$-frames for the wavelet space. 
If the associated frames are bi-orthogonal, we will say that they form bi-orthogonal MRA wavelet families.

5.1. Bi-orthogonal wavelet families. Suppose $\phi_{1}, \ldots, \phi_{d}$ and $\tilde{\phi}_{1}, \ldots, \tilde{\phi}_{d}$ are compactly supported and that they define dual scaling families for an MRA with scaling space $\mathcal{V}$. Now $E=\Lambda \cap \mathcal{V}$ coincides with the compactly supported elements in $\mathcal{V}$. Theorem 4.2 tells us that there exist bi-orthogonal $G$-frames for $\mathcal{V}$ in $\Lambda$. The next theorem tells us in addition that $U \mathcal{V} \cap \Lambda$ contains bi-orthogonal $G$-frames for the wavelet space.

Theorem 5.2. Suppose $\phi_{1}, \ldots, \phi_{d}$ and $\tilde{\phi}_{1}, \ldots, \tilde{\phi}_{d}$ are compactly supported and that they define dual scaling families for an MRA with scaling space $\mathcal{V}$ as in Definition 5.1 .

The wavelet space contains compactly supported bi-orthogonal MRA wavelet families.

Proof. Let $E$ denote the space of compactly supported elements in $\mathcal{V}$, i.e. $E$ is the $\mathcal{A}$-submodule in $\Lambda$ generated by $\phi_{1}, \ldots, \phi_{d}$. The intersection $U \mathcal{V} \cap \Lambda=U E$ defines an $\mathcal{A}$-submodule in $\Lambda$ that contains $E$ as an $\mathcal{A}$-submodule.

By Theorem 4.2, we can choose $\phi_{1}, \ldots, \phi_{d}$ and $\tilde{\phi}_{1}, \ldots, \tilde{\phi}_{d}$ to be bi-orthogonal and dual $G$-frames for $\mathcal{V}$. Now $\phi_{1}, \ldots, \phi_{d}$ and $\tilde{\phi}_{1}, \ldots, \tilde{\phi}_{d}$ form bi-orthogonal $\mathcal{A}$-module frames for $E$.

Let $g_{1}, \ldots, g_{q}$ be a system of representatives of the cosets in $G / A G$. Since $\nu_{g} U=$ $U \nu_{A g}$ for every $g \in G$, we see immediately that $\left\{U \nu_{g_{i}} \phi_{j} \mid 1 \leq i \leq q, 1 \leq j \leq d\right\}$ and $\left\{U \nu_{g_{i}} \tilde{\phi}_{j} \mid 1 \leq i \leq q, 1 \leq j \leq d\right\}$ form bi-orthogonal $G$-frames for the subspace $U \mathcal{V}$. By Corollary [3.5 they also form bi-orthogonal $\mathcal{A}$-module frames for the free $\mathcal{A}$ module $U E$. Let $P \in B\left(L^{2}\left(\mathbb{R}^{n}\right)\right)$ denote the orthogonal projection onto $\mathcal{V}$. We see that

$$
P \zeta=\sum_{i=1}^{d}\left\langle\zeta, \tilde{\phi}_{i}\right\rangle_{\mathcal{A}} \phi_{i}=\sum_{i=1}^{d}\left\langle\zeta, \phi_{i}\right\rangle_{\mathcal{A}} \tilde{\phi}_{i}
$$

for every $\zeta \in \Lambda$. The map $P$ is $\mathcal{A}$-linear, selfadjoint and idempotent on $\Lambda$ such that $P \Lambda=E$. Now $\left\{P U \nu_{g_{i}} \phi_{j} \mid 1 \leq i \leq q, 1 \leq j \leq d\right\}$ and $\left\{P U \nu_{g_{i}} \tilde{\phi}_{j} \mid 1 \leq i \leq q, 1 \leq j \leq d\right\}$ form dual $\mathcal{A}$-module frames for the module $(1-P) U E$. By Lemma 2.1 we see that the submodule $(1-P) U E \subset \Lambda$ is finitely generated, self-dual and projective. Finally, Theorem 4.2 tells us that there exist compactly supported bi-orthogonal $G$-frames $\psi_{1}, \ldots, \psi_{r}$ and $\tilde{\psi}_{1}, \ldots, \tilde{\psi}_{r}$ in $\overline{(1-P) U E}=U \mathcal{V} \ominus \mathcal{V}$.

5.2. Symmetric MRA wavelets. There are several examples of multiresolution analyses in $L^{2}(\mathbb{R})$ generated by dual and compactly supported bi-orthogonal $G$ frames $\phi$ and $\tilde{\phi}$ such that $\phi(x)=\phi(-x) \in \mathbb{R}$ and $\tilde{\phi}(x)=\tilde{\phi}(-x) \in \mathbb{R}$ for almost every $x$. Moreover, these multiresolution analyses allow bi-orthogonal and compactly supported wavelets $\psi$ and $\tilde{\psi}$ such that $\psi\left(x+2^{-1}\right)=\psi\left(-x+2^{-1}\right) \in \mathbb{R}$ and $\tilde{\psi}\left(x+2^{-1}\right)=\tilde{\psi}\left(-x+2^{-1}\right) \in \mathbb{R}$ for almost every $x$; see [Dau92] and [CDF92].

We will be interested in how one can construct bi-orthogonal and compactly supported higher-dimensional MRA wavelets with symmetries if we have compactly supported scaling functions with some symmetry properties. The following definition will turn out to be important. 
Definition 5.3. We will say that a finite subgroup $H \subset G L_{n}(\mathbb{Z})$ is affiliated to the dilation $A$ if it satisfies the following properties:

(1) $h A=A h$,

(2) $(h-I) G \subset A G$,

for every $h \in H$.

Note that if $n=1$ and $H$ is non-trivial, then $A= \pm 2$ and $H=\{ \pm 1\}$ are the only examples. We classified in Røy08 every dilation with a non-trivial affiliated group up to similarity when $n=2$. This classification shows that if $n=2$ and $q=2$, then either $H \simeq \mathbb{Z} / 2 \mathbb{Z}$ or $H \simeq \mathbb{Z} / 4 \mathbb{Z}$. If $H \simeq \mathbb{Z} / 2 \mathbb{Z}$, then $H$ is generated by the matrix $-I_{2}$ and $A$ is similar to one of the following matrices:

$$
\left(\begin{array}{cc}
0 & 2 \\
\pm 1 & 0
\end{array}\right), \pm\left(\begin{array}{cc}
0 & 2 \\
-1 & 1
\end{array}\right), \pm\left(\begin{array}{cc}
1 & -1 \\
1 & 1
\end{array}\right)
$$

Moreover, if $H \simeq \mathbb{Z} / 4 \mathbb{Z}$ and $h$ generates $H$, then there exists an $S \in G L_{2}(\mathbb{Z})$ such that

$$
S h S^{-1}=\left(\begin{array}{cc}
0 & 1 \\
-1 & 0
\end{array}\right) \text { and } S A S^{-1}= \pm\left(\begin{array}{cc}
1 & -1 \\
1 & 1
\end{array}\right)
$$

Theorem 5.4. Suppose $q=2$ and suppose $\phi$ and $\tilde{\phi}$ have compact supports and generate an MRA with dilation $A$ and scaling space $\mathcal{V}$. If $g_{1} \in G \backslash A G$, then the vectors

$$
\begin{aligned}
\psi & =\left\langle U \nu_{g_{1}} \phi, \tilde{\phi}\right\rangle_{\mathcal{A}} U \phi-\langle U \phi, \tilde{\phi}\rangle_{\mathcal{A}} U \nu_{g_{1}} \phi \\
\tilde{\psi} & =\left\langle U \nu_{g_{1}} \tilde{\phi}, \phi\right\rangle_{\mathcal{A}} U \tilde{\phi}-\langle U \tilde{\phi}, \phi\rangle_{\mathcal{A}} U \nu_{g_{1}} \tilde{\phi}
\end{aligned}
$$

define bi-orthogonal MRA wavelets in $\Lambda \cap U \mathcal{V}$. Moreover, if $H$ is affiliated to $A$ and $\phi(h x)=\phi(x)$ and $\tilde{\phi}(h x)=\tilde{\phi}(x)$ for every $h \in H$ and a.e. $x \in \mathbb{R}^{n}$, then

$$
\psi\left(h x+A^{-1} g_{1}\right)=\psi\left(x+A^{-1} g_{1}\right) \text { and } \tilde{\psi}\left(h x+A^{-1} g_{1}\right)=\tilde{\psi}\left(x+A^{-1} g_{1}\right)
$$

for every $h \in H$ and a.e. $x \in \mathbb{R}^{n}$.

Proof. First, let $E$ denote the $\mathcal{A}$-module $\Lambda \cap \mathcal{V}$. Note that $U \zeta \in \Lambda$ if and only if $U \zeta \in \Lambda$. This and the assumption $\mathcal{V} \subset U \mathcal{V}$ imply that $E \subset U E \subset \Lambda$ and $U \mathcal{V} \cap \Lambda=U E$. We have that $\left\{U \nu_{g} \phi\right\}_{g \in G}$ and $\left\{U \nu_{g} \tilde{\phi}\right\}_{g \in G}$ form bi-orthogonal frames for the space $U \mathcal{V}$. The element $g_{1}$ represents the only non-trivial coset in $G$. Since $\nu_{g} U=\nu_{A g}$ for every $g \in G$, we see that $\left\{\nu_{g} U \nu_{f} \phi \mid f \in\left\{0, g_{1}\right\}, g \in G\right\}$ and $\left\{\nu_{g} U \nu_{f} \tilde{\phi} \mid f \in\left\{0, g_{1}\right\}, g \in G\right\}$ form bi-orthogonal frames for $U \mathcal{V}$. Now $\left\{U \phi, U \nu_{g_{1}} \phi\right\}$ and $\left\{U \tilde{\phi}, U \nu_{g_{1}} \tilde{\phi}\right\}$ form dual module frames for the $\mathcal{A}$-module $U E$. To simplify our notation slightly, we let $\zeta_{1}=U \phi, \zeta_{2}=U \nu_{g_{1}} \phi, \tilde{\zeta}_{1}=U \tilde{\phi}$ and $\tilde{\zeta}_{2}=U \nu_{g_{1}} \tilde{\phi}$.

We will first check that $\{\phi, \psi\}$ and $\{\tilde{\phi}, \tilde{\psi}\}$ form dual module frames for $U E$ : Since $\left\{\zeta_{1}, \zeta_{2}\right\}$ and $\left\{\tilde{\zeta}_{1}, \tilde{\zeta}_{2}\right\}$ form dual module bases for $U E$, we obtain the following identities:

$$
\begin{aligned}
& \left\langle\tilde{\zeta}_{1}, \psi\right\rangle_{\mathcal{A}}\left\langle\tilde{\psi}, \zeta_{1}\right\rangle_{\mathcal{A}}=\left\langle\tilde{\phi}, \zeta_{2}\right\rangle_{\mathcal{A}}\left\langle\tilde{\zeta}_{2}, \phi\right\rangle_{\mathcal{A}}, \\
& \left\langle\tilde{\zeta}_{1}, \psi\right\rangle_{\mathcal{A}}\left\langle\tilde{\psi}, \zeta_{2}\right\rangle_{\mathcal{A}}=-\left\langle\tilde{\zeta}_{1}, \phi\right\rangle_{\mathcal{A}}\left\langle\tilde{\phi}, \zeta_{2}\right\rangle_{\mathcal{A}}, \\
& \left\langle\tilde{\zeta}_{2}, \psi\right\rangle_{\mathcal{A}}\left\langle\tilde{\psi}, \zeta_{1}\right\rangle_{\mathcal{A}}=-\left\langle\tilde{\zeta}_{2}, \phi\right\rangle_{\mathcal{A}}\left\langle\tilde{\phi}, \zeta_{1}\right\rangle_{\mathcal{A}}, \\
& \left\langle\zeta_{2}, \psi\right\rangle_{\mathcal{A}}\left\langle\tilde{\psi}, \zeta_{2}\right\rangle_{\mathcal{A}}=\left\langle\tilde{\zeta}_{1}, \phi\right\rangle_{\mathcal{A}}\left\langle\tilde{\phi}, \zeta_{1}\right\rangle_{\mathcal{A}} .
\end{aligned}
$$


Putting these together, we see that

$$
\left\langle\tilde{\zeta}_{i}, \phi\right\rangle_{\mathcal{A}}\left\langle\tilde{\phi}, \zeta_{j}\right\rangle_{\mathcal{A}}+\left\langle\tilde{\zeta}_{i}, \psi\right\rangle_{\mathcal{A}}\left\langle\tilde{\psi}, \zeta_{j}\right\rangle_{\mathcal{A}}=\delta_{i, j}
$$

If we let $\zeta, \eta \in U E$ and apply (5.2), then we obtain

$$
\begin{aligned}
& \langle\zeta, \phi\rangle_{\mathcal{A}}\langle\tilde{\phi}, \eta\rangle_{\mathcal{A}}+\langle\zeta, \psi\rangle_{\mathcal{A}}\langle\tilde{\psi}, \eta\rangle_{\mathcal{A}} \\
= & \sum_{i, j}\left\langle\zeta, \zeta_{i}\right\rangle_{\mathcal{A}}\left\langle\tilde{\zeta}_{i}, \phi\right\rangle_{\mathcal{A}}\left\langle\tilde{\phi}, \zeta_{j}\right\rangle_{\mathcal{A}}\left\langle\tilde{\zeta}_{j}, \eta\right\rangle_{\mathcal{A}}+\sum_{i, j}\left\langle\zeta, \zeta_{i}\right\rangle_{\mathcal{A}}\left\langle\tilde{\zeta}_{i}, \psi\right\rangle_{\mathcal{A}}\left\langle\tilde{\psi}, \zeta_{j}\right\rangle_{\mathcal{A}}\left\langle\tilde{\zeta}_{j}, \eta\right\rangle_{\mathcal{A}} \\
= & \sum_{i, j}\left\langle\zeta, \zeta_{i}\right\rangle_{\mathcal{A}}\left(\left\langle\tilde{\zeta}_{i}, \phi\right\rangle_{\mathcal{A}}\left\langle\tilde{\phi}, \zeta_{j}\right\rangle_{\mathcal{A}}+\left\langle\tilde{\zeta}_{i}, \psi\right\rangle_{\mathcal{A}}\left\langle\tilde{\psi}, \zeta_{j}\right\rangle_{\mathcal{A}}\right)\left\langle\tilde{\zeta}_{j}, \eta\right\rangle_{\mathcal{A}} \\
= & \sum_{i}\left\langle\zeta, \zeta_{i}\right\rangle_{\mathcal{A}}\left\langle\tilde{\zeta}_{i}, \eta\right\rangle_{\mathcal{A}}=\langle\zeta, \eta\rangle_{\mathcal{A}},
\end{aligned}
$$

so $\{\phi, \psi\}$ and $\{\tilde{\phi}, \tilde{\psi}\}$ form dual module frames for $U E$.

Next, we compute that

$$
\langle\phi, \tilde{\psi}\rangle_{\mathcal{A}}=\left\langle\phi, \tilde{\zeta}_{1}\right\rangle_{\mathcal{A}}\left\langle\phi, \tilde{\zeta}_{2}\right\rangle_{\mathcal{A}}-\left\langle\phi, \tilde{\zeta}_{2}\right\rangle_{\mathcal{A}}\left\langle\phi, \tilde{\zeta}_{1}\right\rangle_{\mathcal{A}}=0 .
$$

An equivalent computation shows that $\langle\tilde{\phi}, \psi\rangle_{\mathcal{A}}=0$. Finally, we compute that

$$
\begin{aligned}
\langle\psi, \tilde{\psi}\rangle_{\mathcal{A}} & =\left\langle\left\langle\zeta_{2}, \tilde{\phi}\right\rangle_{\mathcal{A}} \zeta_{1}-\left\langle\zeta_{1}, \tilde{\phi}\right\rangle_{\mathcal{A}} \zeta_{2},\left\langle\tilde{\zeta}_{2}, \phi\right\rangle_{\mathcal{A}} \tilde{\zeta}_{1}-\left\langle\tilde{\zeta}_{1}, \phi\right\rangle_{\mathcal{A}} \tilde{\zeta}_{2}\right\rangle_{\mathcal{A}} \\
& =\sum_{i}\left\langle\phi, \tilde{\zeta}_{i}\right\rangle_{\mathcal{A}}\left\langle\zeta_{i}, \tilde{\phi}\right\rangle_{\mathcal{A}}=\langle\phi, \tilde{\phi}\rangle_{\mathcal{A}}=1,
\end{aligned}
$$

so $\{\phi, \psi\}$ and $\{\tilde{\phi}, \tilde{\psi}\}$ form dual module bases for $U E$. By Corollary 3.5 , we see that $\psi$ and $\tilde{\psi}$ form bi-orthogonal and compactly supported MRA wavelets in $U E$. For every $h \in H$, we define a unitary operator on $L^{2}\left(\mathbb{R}^{n}\right)$ with the following equation: $W_{h} \xi(x)=\xi\left(h^{-1} x\right)$. These unitaries define a unitary representation of $H$ on $L^{2}\left(\mathbb{R}^{n}\right)$. Whenever $\eta_{1}, \eta_{2}, \zeta \in \Lambda$, then

$$
\begin{aligned}
\left\langle W_{h} \eta_{1}, W_{h} \eta_{2}\right\rangle_{\mathcal{A}} \zeta & =\sum_{g \in G}\left\langle W_{h} \eta_{1}, \nu_{g} W_{h} \eta_{2}\right\rangle \nu_{g} \zeta=\sum_{g \in G}\left\langle\eta_{1}, W_{h}^{-1} \nu_{g} W_{h} \eta_{2}\right\rangle \nu_{g} \zeta \\
& =\sum_{g \in G}\left\langle\eta_{1}, \nu_{g} \eta_{2}\right\rangle W_{h} \nu_{g} W_{h}^{-1} \zeta=W_{h}\left\langle\eta_{1}, \eta_{2}\right\rangle_{\mathcal{A}} W_{h}^{-1} \zeta .
\end{aligned}
$$

We have that $W_{h} U=U W_{h}$ for every $h \in H$, since $h A=A h$ for every $h \in H$. Moreover, since $(h-1) G \subset A G$ for every $h \in H$, there exists a $g_{h} \in G$ for every $h \in H$ s.t.

$$
U W_{h} \nu_{g_{1}} W_{h}^{-1} \nu_{g_{1}}^{-1} U^{-1}=\nu_{g_{h}} .
$$

We also see that $U \nu_{g_{1}} U^{-1}$ commutes with every element in $\pi_{0}(\mathcal{A})$. Now

$$
\begin{aligned}
W_{h} U \nu_{g_{1}}^{-1} U^{-1} \psi & =W_{h} U \nu_{g_{1}}^{-1} U^{-1}\left\langle U \nu_{g_{1}} \phi, \tilde{\phi}\right\rangle_{\mathcal{A}} U \phi-W_{h} U \nu_{g}^{-1} U^{-1}\langle U \phi, \tilde{\phi}\rangle_{\mathcal{A}} U \nu_{g_{1}} \phi \\
& =W_{h} U \nu_{g_{1}}^{-1} U^{-1} W_{h}^{-1} W_{h}\left\langle U \nu_{g_{1}} \phi, \tilde{\phi}\right\rangle_{\mathcal{A}} W_{h}^{-1} U \phi-W_{h}\langle U \phi, \tilde{\phi}\rangle_{\mathcal{A}} U \phi \\
& =W_{h} U \nu_{g_{1}}^{-1} U^{-1} W_{h}^{-1}\left\langle U W_{h} \nu_{g_{1}} W_{h}^{-1} \phi, \tilde{\phi}\right\rangle_{\mathcal{A}} U \phi-W_{h}\langle U \phi, \tilde{\phi}\rangle_{\mathcal{A}} W_{h}^{-1} U \phi \\
& =W_{h} U \nu_{g_{1}}^{-1} U^{-1} W_{h}^{-1}\left\langle\nu_{g_{h}} U \nu_{g_{1}} \phi, \tilde{\phi}\right\rangle_{\mathcal{A}} U \phi-\left\langle W_{h} U \phi, W_{h} \tilde{\phi}\right\rangle_{\mathcal{A}} U \phi \\
& =W_{h} U \nu_{g_{1}}^{-1} U^{-1} W_{h}^{-1} \nu_{g_{h}}\left\langle U \nu_{g_{1}} \phi, \tilde{\phi}\right\rangle_{\mathcal{A}} U \phi-\langle U \phi, \tilde{\phi}\rangle_{\mathcal{A}} U \phi \\
& =U \nu_{g_{1}}^{-1} U^{-1}\left\langle U \nu_{g_{1}} \phi, \tilde{\phi}\right\rangle_{\mathcal{A}} U \phi-\langle U \phi, \tilde{\phi}\rangle_{\mathcal{A}} U \phi \\
& =U \nu_{g_{1}}^{-1} U^{-1}\left(\left\langle U \nu_{g_{1}} \phi, \tilde{\phi}\right\rangle_{\mathcal{A}} U \phi-\langle U \phi, \tilde{\phi}\rangle_{\mathcal{A}} U \nu_{g_{1}} \phi\right)=U \nu_{g_{1}}^{-1} U^{-1} \psi,
\end{aligned}
$$


i.e. $\psi\left(h x+A^{-1} g_{1}\right)=\psi\left(x+A^{-1} g_{1}\right)$ for almost every $x \in \mathbb{R}^{n}$. The analogous statement about $\tilde{\psi}$ follows from an identical computation.

\section{ACKNOWLEDGEMENTS}

The author is pleased to acknowledge helpful discussions with Ola Bratteli and Martin Gulbrandsen.

\section{REFERENCES}

[BR87] Ola Bratteli and Derek W. Robinson. Operator algebras and quantum statistical mechanics. 1. Texts and Monographs in Physics. Springer-Verlag, New York, second edition, 1987. $C^{*}$ - and $W^{*}$-algebras, symmetry groups, decomposition of states. MR 887100 (88d:46105)

[CDF92] A. Cohen, Ingrid Daubechies, and J.-C. Feauveau. Biorthogonal bases of compactly supported wavelets. Comm. Pure Appl. Math., 45(5):485-560, 1992. MR.1162365 (93e:42044)

[Dau92] Ingrid Daubechies. Ten lectures on wavelets, volume 61 of CBMS-NSF Regional Conference Series in Applied Mathematics. Society for Industrial and Applied Mathematics (SIAM), Philadelphia, PA, 1992. MR1162107 (93e:42045)

[Dav96] Kenneth R. Davidson. $C^{*}$-algebras by example, volume 6 of Fields Institute Monographs. American Mathematical Society, Providence, RI, 1996. MR.1402012 (97i:46095)

[DR07] Dorin Ervin Dutkay and Kjetil Røysland. The algebra of harmonic functions for a matrix-valued transfer operator. J. Funct. Anal., 252(2):734-762, 2007. MR2360935 $(2008 \mathrm{~m}: 42056)$

[FL02] Michael Frank and David R. Larson. Frames in Hilbert $C^{*}$-modules and $C^{*}$-algebras. $J$. Operator Theory, 48(2):273-314, 2002. MR1938798 (2003i:42040)

[Gub88] I. Dzh. Gubeladze. The Anderson conjecture and a maximal class of monoids over which projective modules are free. Mat. Sb. (N.S.), 135(177)(2):169-185, 271, 1988. MR937805 (89d:13010)

[HL00] Deguang Han and David R. Larson. Frames, bases and group representations. Mem. Amer. Math. Soc., 147(697):x+94, 2000. MR1686653 (2001a:47013)

[Lam78] T. Y. Lam. Serre's conjecture. Lecture Notes in Mathematics, Vol. 635. Springer-Verlag, Berlin, 1978. MR0485842(58:5644)

[Lam06] T. Y. Lam. Serre's problem on projective modules. Springer Monographs in Mathematics. Springer-Verlag, Berlin, 2006. MR2235330 (2007b:13014)

[Lan95] E. C. Lance. Hilbert $C^{*}$-modules, volume 210 of London Mathematical Society Lecture Note Series. Cambridge University Press, Cambridge, 1995. A toolkit for operator algebraists. MR 1325694 (96k:46100)

[Lan02] Serge Lang. Algebra, volume 211 of Graduate Texts in Mathematics. Springer-Verlag, New York, third edition, 2002. MR.1878556 (2003e:00003)

[LR07] Nadia S. Larsen and Iain Raeburn. Projective multi-resolution analyses arising from direct limits of Hilbert modules. Math. Scand., 100(2):317-360, 2007. MR2339372 (2008m:42059)

[Pac07] Judith A. Packer. Projective multiresolution analyses for dilations in higher dimensions. J. Operator Theory, 57(1):147-172, 2007. MR2304920(2008b:46098)

[Ped89] Gert K. Pedersen. Analysis now, volume 118 of Graduate Texts in Mathematics. Springer-Verlag, New York, 1989. MR971256 (90f:46001)

[PR03] Judith A. Packer and Marc A. Rieffel. Wavelet filter functions, the matrix completion problem, and projective modules over $C\left(\mathbb{T}^{n}\right)$. J. Fourier Anal. Appl., 9(2):101-116, 2003. MR:1964302 (2003m:42063)

[PR04] Judith A. Packer and Marc A. Rieffel. Projective multi-resolution analyses for $L^{2}\left(\mathbb{R}^{2}\right)$. J. Fourier Anal. Appl., 10(5):439-464, 2004. MR2093911 (2005f:46133)

[Røy08] Kjetil Røysland. Symmetries in projective multiresolution analyses. J. Fourier Anal. Appl., 14(2):267-285, 2008. MR2383725 (2009i:42016) 
[Swa78] Richard G. Swan. Projective modules over Laurent polynomial rings. Trans. Amer. Math. Soc., 237:111-120, 1978. MR0469906 (57:9686)

[Woo04] Peter John Wood. Wavelets and Hilbert modules. J. Fourier Anal. Appl., 10(6):573-598, 2004. MR2105534 (2005h:42079)

Department of Mathematics, University of Oslo, PO Box 1053, Blindern, NO-0316 OSLO, NORWAY

Current address: Department of Biostatistics, University of Oslo, Sognsvannsv. 9, PO Box 1122, Blindern, NO-0317 Oslo, Norway

E-mail address: roysland@math.uio.no 